\title{
Availability Equivalence Factors of a General Repairable Parallel-Series System
}

\author{
Abdelfattah Mustafa', Ammar M. Sarhan² \\ ${ }^{1}$ Department of Mathematics, Faculty of Science, Mansoura University, Mansoura, Egypt \\ ${ }^{2}$ Department of Mathematics \& Statistics, Dalhousie University, Nova Scotia, Canada \\ Email: abdelfatah mustafa@yahoo.com, asarhan@yahoo.com
}

Received 21 February 2014; revised 1 April 2014; accepted 8 April 2014

Copyright $(02014$ by authors and Scientific Research Publishing Inc.

This work is licensed under the Creative Commons Attribution International License (CC BY). http://creativecommons.org/licenses/by/4.0/

(c) ()

\begin{abstract}
The availability equivalence factors of a general repairable parallel-series system are discussed in this paper considering the availability function of the system. The system components are assumed to be repairable and independent but not identical. The life and repair times of the system components are exponentially distributed with different parameters. Two types of availability equivalent factors of the system are derived. The results derived in this paper generalize those given in the literature. A numerical example is introduced to illustrate how the idea of this work can be applied.
\end{abstract}

Keywords

Reliability Engineering, Duplication Methods, Repairable Systems

\section{Introduction}

In reliability analysis, there are two main methods to improve non-repairable system design. These methods are the reduction and redundancy methods [1]. In the reduction method, it is assumed that the system design can be improved by reducing the failure rate(s) of a set of system components by a factor $\rho, 0<\rho<1$, [1]-[4]. The redundancy method assumes that the system can be improved by increasing its components [5].

There are more than one redundancy methods such as hot, warm, cold and cold with imperfect switch redundancy, named respectively as hot, warm, cold and cold with imperfect switch duplication methods [6]. The redundancy methods can be applied on repairable systems as well. In addition to the reduction method, the repairable system can be improved by increasing the repair rate of some of the system component(s) by a factor $\sigma$, $\sigma>1,[7][8]$.

Using the redundancy method may not be a practical solution for a system in which the minimum size and 
weight are overriding considerations: for example, in satellites or other space applications, in well-logging equipment, and in pacemakers and similar biomedical applications [9]. In such applications space or weight limitations may indicate an increase in component performance rather than redundancy. Then more emphasis must be placed on better design, manufacturing quality control and on controlling the operating environment. Therefore, the concept of reliability/availability equivalence takes place. In such concept, the design of the system that is improved according to reduction or increasing method should be equivalent to the design of the system improved according to one of redundancy methods. That is, in this concept, one may say that the performance of a system can be improved through an alternative design [10]. In this case, different system designs should be comparable based on a performance characteristic such as 1) the reliability function or mean time to failure in the case of no repairs or 2) the availability in the case of repairable systems.

The concept of comparing different designs is applied in the literature in order to: 1) improve the reliability of a non-repairable system [11]; 2) determine a representative service provider and create equivalent elements [12]; 3) derive the reliability equivalence factors of some non-repairable systems [2] and the references therein; and 4) derive the availability equivalence factors of a repairable system [7] [8].

The reliability equivalence concept applied on various non-repairable systems, [1] [2] [4] [13]-[17].

In this work, the reliability function and mean time to failure are used as characteristic measures to compare different system designs to derive reliability/mean time equivalence factors.

Repairable system indicates a system that can be repaired to operate normally in the event of any failure, such as automobiles, airplanes, computer network, manufacturing system, sewage systems, power plant or fire prevention system. Availability comprises "reliability" and "recovery part of unreliability after repair", indicating the probability that repairable systems, machines or components maintain the function at a specific moment [18]. It is generally expressed as the operable time over total time. Parallel-series system indicates sub-systems in which several components are connected in series, and then in parallel, or sub-systems that several components are connected in parallel, and then in series [19]. The reliability/availability of a parallel-series system has drawn continuous attention in both problem characteristics and solution methodologies [2], [19] and [20]. Recently, [7] [8] discussed the availability equivalence factors of a repairable series-parallel system with independent and identical (non-identical) components.

Our goal in this paper is to derive the availability equivalence factors of a repairable parallel-series system with independent and non-identical components. The availability function of the system will be used as a performance measure to compare different system designs of the original system and other improved systems in order to derive these factors.

The structure of this paper is organized as follows. Section 2 introduces the illustration of the parallel-series system and the system availability. Section 3 presents the availabilities of the systems improved according to five different methods that can be applied to improve the performance of the original system. In Section 4, two types of availability equivalence factors of the system are discussed. A numerical example is introduced in Section 5 to illustrate how the idea of this work can be applied. Finally, Section 6 is devoted to the conclusions, which handle the main results derived throughout this work.

\section{A General Repairable Parallel-Series System}

The system considered here consists of $n$ subsystems connected in parallel, and with subsystem $i$ consisting of $m_{i}$ independent, repairable and nonidentical components connected in series for $i=1,2, \cdots, n$. We refer to such system as a general repairable parallel-series system. Figure 1 shows the diagram of that system.

Let $T_{i j}$ and $Y_{i j}$ be the lifetime and repair time, respectively, of component $j$ in subsystem $i, 1 \leq i \leq n$, $1 \leq j \leq m_{i}$. It is assumed that the life and repair times of component $j$ in subsystem $i, 1 \leq i \leq n, 1 \leq j \leq m_{i}$, follow exponential distributions with failure rate $\lambda_{i j}$ and repair rate $\mu_{i j}$. Let $N$ be the total number of the system components, that is $N=\sum_{i=1}^{n} m_{i}$.

Special Cases: This system generalizes the following cases:

1) Repairable parallel-series system with identical components, when $\lambda_{i j}=\lambda, \mu_{i j}=\mu, j=1,2, \cdots, m_{i}$ and $i=1,2, \cdots, n$.

2) Repairable parallel system with non-identical components, when $m_{i}=1$ and $i=1,2, \cdots, n$.

3) Repairable series system with non-identical components, when $n=1$ and $j=1,2, \cdots, m$.

Let $A_{i j}$, be the availability of the component $j$ in subsystem $i$ and $A_{i}$ be the availability of the subsystem $i$, 


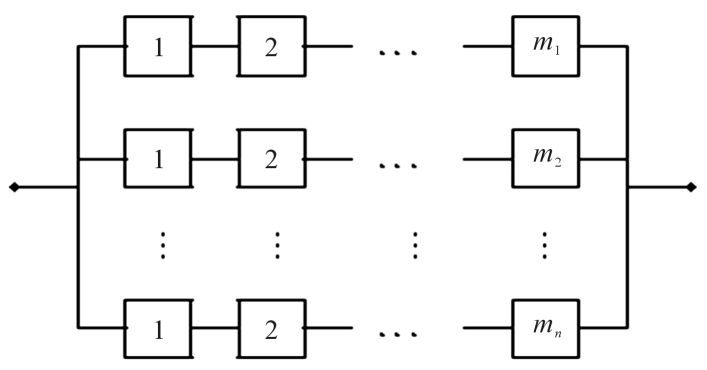

Figure 1. Parallel-series system structure.

$1 \leq i \leq n, \quad 1 \leq j \leq m_{i}$. One can easily derive $A_{i j}$ and $A_{i}$, respectively, as, see [8]

and

$$
A_{i j}=\frac{\mu_{i j}}{\mu_{i j}+\lambda_{i j}}=\frac{1}{1+\eta_{i j}} \text {, where } \eta_{i j}=\frac{\lambda_{i j}}{\mu_{i j}},
$$

$$
A_{i}=\prod_{j=1}^{m_{i}} A_{i j}=\prod_{j=1}^{m_{i}}\left(\frac{1}{1+\eta_{i j}}\right) .
$$

Therefore, the system availability, denoted $A_{s}$, can be derived as

$$
A_{s}=1-\prod_{i=1}^{n}\left(1-A_{i}\right)=1-\prod_{i=1}^{n}\left[1-\prod_{j=1}^{m_{i}}\left(\frac{1}{1+\eta_{i j}}\right)\right] .
$$

\section{Different Designs of Improved System}

The system can be improved according to one of the following three different methods:

1) Reduction method. In this method it is assumed that the component can be improved by reducing its failure rate by a factor $\rho, 0<\rho<1$.

2) Increasing method. It is assumed in this method that the component can be improved by increasing its repair rate by a factor $\sigma, \sigma>1$.

3) Standby redundancy method:

a) Hot duplication method: in this method we assume that the component is duplicated by an identical hot standby component.

b) Warm duplication method: in this method we assume that the component is duplicated by an identical warm standby component.

c) Cold duplication method: in this method we assume that the component is duplicated by an identical cold standby component.

In the following sections, we derive the availability of the system improved according to the methods mentioned above.

\subsection{The Reduction Method}

It is assumed in the reduction method that the system can be improved by reducing the failure rates of a set $R$ components by a factor $\rho, 0<\rho<1$. We assume that $R=\bigcup_{i=1}^{n} R_{i}$, where $R_{i}$ is a set of the subsystem $i$ components, $1 \leq i \leq n$. Also, we assume that $\left|R_{i}\right|=r_{i}, \quad 0 \leq r_{i} \leq m_{i}$, and $|R|=r=\sum_{i=1}^{n} r_{i},(1 \leq r \leq N)$.

Let $A_{i j, \rho}$ be the availability of the component $j$ in subsystem $i$, improved by reducing its failure rate $\lambda_{i j}$ by the factor $\rho$. One can easily derive

$$
A_{i j, \rho}=\frac{1}{1+\rho \eta_{i j}} \text {, where } \eta_{i j}=\frac{\lambda_{i j}}{\mu_{i j}} .
$$

Therefore, the availability of subsystem $i$ improved by reducing the failure rates of a set $R_{i}$ components by the factor $\rho$, denoted $A_{R_{i}, \rho}$, can be written as 


$$
A_{R_{i}, \rho}=\prod_{j \in R_{i}} A_{i j, \rho} \prod_{j \in \bar{R}_{i}} A_{i j}=\prod_{j \in R_{i}}\left(\frac{1}{1+\rho \eta_{i j}}\right) \prod_{j \in \bar{R}_{i}}\left(\frac{1}{1+\eta_{i j}}\right),
$$

where $\bar{R}_{i}=M_{i} \backslash R_{i}, \quad M_{i}$ is the set of all subsystem $i$ components, $M_{i}=\left\{1,2, \cdots, m_{i}\right\}, 1 \leq i \leq n$.

Finally, the availability of the system improved by reducing the failure rates of a set $R$ components by the same factor $\rho$, denoted $A_{R, \rho}$, can be derived as

$$
A_{R, \rho}=1-\prod_{i=1}^{n}\left[1-\prod_{j \in R_{i}}\left(\frac{1}{1+\rho \eta_{i j}}\right) \prod_{j \in \bar{R}_{i}}\left(\frac{1}{1+\eta_{i j}}\right)\right] .
$$

\subsection{The Increasing Method}

It is assumed in the increasing method that the system can be improved by increasing the repair rates of a set $S$ components by a factor $\sigma, \sigma>1$. We assume that $S=\bigcup_{i=1}^{n} S_{i}$, where $S_{i}$ is a set of the subsystem $i$ components, $1 \leq i \leq n$. Also, we assume that $\left|S_{i}\right|=s_{i}, 0 \leq s_{i} \leq m_{i}$, and $|S|=s=\sum_{i=1}^{n} s_{i}, \quad 1 \leq s \leq N$.

Let $A_{i j, \sigma}$ be the availability of component $j$ in subsystem $i$ after increasing its repair rate $\mu_{i j}$ by the factor $\sigma, \sigma>1$ and $A_{s_{i}, \sigma}$ be the availability of subsystem $i$ which is improved by increasing the repair rates of a set $S_{i}$ components by the same factor $\sigma$; and $A_{S, \sigma}$ be the availability of the system improved by increasing the repair rates of a set $S$ components by the same factor $\sigma$. One can derive these availabilities in the following forms

$$
\begin{gathered}
A_{i j, \sigma}=\frac{\sigma \mu_{i j}}{\sigma \mu_{i j}+\lambda_{i j}}=\frac{\sigma}{\sigma+\eta_{i j}}, \\
A_{S_{i}, \sigma}=\prod_{j \in S_{i}} A_{i j, \sigma} \prod_{j \in \bar{S}_{i}} A_{i j}=\prod_{j \in S_{i}}\left(\frac{\sigma}{\sigma+\eta_{i j}}\right) \prod_{j \in \bar{S}_{i}}\left(\frac{1}{1+\eta_{i j}}\right), \\
A_{S, \sigma}=1-\prod_{i=1}^{n}\left[1-\prod_{j \in S_{i}}\left(\frac{\sigma}{\sigma+\eta_{i j}}\right) \prod_{j \in \bar{S}_{i}}\left(\frac{1}{1+\eta_{i j}}\right)\right],
\end{gathered}
$$

where $\bar{S}_{i}=M \backslash S_{i}$, for $1 \leq i \leq n$.

\subsection{The Hot Duplication Method}

It is assumed in the hot duplication method that the system can be improved by connecting every element in a set $B$ components with an identical component in parallel. We assume that $B=\bigcup_{i=1}^{n} B_{i}$, where $B_{i}$ is a set of the subsystem $i$ components, $1 \leq i \leq n$. Also, we assume that $\left|B_{i}\right|=h_{i}, 0 \leq h_{i} \leq m_{i}$, and $|B|=h=\sum_{i=1}^{n} h_{i}, 1 \leq h \leq N$.

Let $A_{B_{i}}^{H}$ be the availability of the subsystem $i$ which is improved by improving a set $B_{i} \subseteq M_{i}$ components, $1 \leq i \leq n$; and $A_{B}^{H}$ be the availability of the system improved by improving a set $B$ components according to the hot duplication method. One can derive

$$
\begin{gathered}
A_{B_{i}}^{H}=\prod_{j \in B_{i}}\left[1-\left(1-A_{i j}\right)^{2}\right] \prod_{j \in \bar{B}_{i}} A_{i j}=\prod_{j \in B_{i}}\left[1-\left(\frac{\eta_{i j}}{1+\eta_{i j}}\right)^{2}\right] \prod_{j \in \bar{B}_{i}}\left(\frac{1}{1+\eta_{i j}}\right), \\
A_{B}^{H}=1-\prod_{i=1}^{n}\left[1-\prod_{j \in B_{i}}\left[1-\left(\frac{\eta_{i j}}{1+\eta_{i j}}\right)^{2}\right] \prod_{j \in \bar{B}_{i}}\left(\frac{1}{1+\eta_{i j}}\right)\right],
\end{gathered}
$$

where $\bar{B}_{i}=M_{i} \backslash B_{i}$, for $1 \leq i \leq n$.

\subsection{The Warm Duplication Method}

We say that, a component $j$ in subsystem $i$ is warm duplicated if it is connected in parallel with a non-identical component, having a failure rate $v_{i j}$, in parallel via a perfect switch. In the warm duplication method, it is as- 
sumed that the system can be improved when every component in a set $B$ components is warm duplicated. We assume that $B=\bigcup_{i=1}^{n} B_{i}$, where $B_{i}$ is a set of the subsystem $i$ components, $1 \leq i \leq n$. Also, we assume that $\left|B_{i}\right|=w_{i}, \quad 0 \leq w_{i} \leq m_{i}$, and $|B|=w=\sum_{i=1}^{n} w_{i}, 1 \leq w \leq N$.

Let $A_{i j}^{W}$ be the availability of the component $j$ in the subsystem $i$ when it is improved according to the warm duplication method. Using Markov process, $A_{i j}^{W}$ can be obtained as follows, see [21],

$$
A_{i j}^{W}=\frac{1+\eta_{i j}+\xi_{i j}}{1+\eta_{i j}+\xi_{i j}+\frac{1}{2} \eta_{i j}^{2}+\frac{1}{2} \eta_{i j} \xi_{i j}},
$$

where $\xi_{i j}=v_{i j} / \mu_{i j}$, for $1 \leq j \leq m_{i}$ and $1 \leq i \leq n$.

Let $A_{B_{i}}^{W}$ be the availability of the subsystem $i$ improved by improving $B_{i}$ subsystem components according to the warm duplication method. Therefore, one can derive

$$
A_{B_{i}}^{W}=\prod_{j \in B_{i}}\left(\frac{1+\eta_{i j}+\xi_{i j}}{1+\eta_{i j}+\xi_{i j}+\frac{1}{2} \eta_{i j}^{2}+\frac{1}{2} \eta_{i j} \xi_{i j}}\right) \prod_{j \in \bar{B}_{i}}\left(\frac{1}{1+\eta_{i j}}\right),
$$

Finally, let $A_{B}^{W}$ be the availability of the system improved by improving a set $B$ components according to the warm duplication methods. Using Equation (13), we get

$$
A_{B}^{W}=1-\prod_{i=1}^{n}\left[1-\prod_{j \in B_{i}}\left(\frac{1+\eta_{i j}+\xi_{i j}}{1+\eta_{i j}+\xi_{i j}+\frac{1}{2} \eta_{i j}^{2}+\frac{1}{2} \eta_{i j} \xi_{i j}}\right) \prod_{j \in \bar{B}_{i}}\left(\frac{1}{1+\eta_{i j}}\right)\right] .
$$

\subsection{The Cold Duplication Method}

It is assumed in the cold duplication method, that each component of set $B$ components is connected in parallel with an identical component via a perfect switch. We assume that $B=\bigcup_{i=1}^{n} B_{i}$, where $B_{i}$ is a set of the subsystem $i$ components, $1 \leq i \leq n$. Also, we assume that $\left|B_{i}\right|=c_{i}, \quad 0 \leq c_{i} \leq m_{i}$, and $|B|=c=\sum_{i=1}^{n} c_{i}, 1 \leq c \leq N$.

Let $A_{i j}^{C}$ is the availability of the component $j$ in subsystem $i$ when it is improved according to the cold duplication method; $A_{B_{i}}^{C}$ be the availability of subsystem $i$, which is improved according to cold duplication method; and $A_{B}^{C}$ be the availability of the system improved by improving set $B$ components according to the cold duplication method. Using Markov process theory, $A_{i j}^{C}$ is, see [22],

$$
A_{i j}^{C}=\frac{\mu_{i j}^{2}+\lambda_{i j} \mu_{i j}}{\mu_{i j}^{2}+\lambda_{i j} \mu_{i j}+\frac{1}{2} \lambda_{i j}^{2}}=\frac{1+\eta_{i j}}{1+\eta_{i j}+\frac{1}{2} \eta_{i j}^{2}} .
$$

Using Equation (15) and the nature of the series subsystem $i$, one can derive

$$
A_{B_{i}}^{C}=\prod_{j \in B_{i}}\left(\frac{1+\eta_{i j}}{1+\eta_{i j}+\frac{1}{2} \eta_{i j}^{2}}\right) \prod_{j \in \bar{B}_{i}}\left(\frac{1}{1+\eta_{i j}}\right) .
$$

Finally, using Equation (16) and the nature of the parallel connection of the subsystems, we get

$$
A_{B}^{C}=1-\prod_{i=1}^{n}\left[1-\prod_{j \in B_{i}}\left(\frac{1+\eta_{i j}}{1+\eta_{i j}+\frac{1}{2} \eta_{i j}^{2}}\right) \prod_{j \in \bar{B}_{i}}\left(\frac{1}{1+\eta_{i j}}\right)\right] .
$$

\section{Availability Equivalence Factors}

In this section, we derive the availability equivalence factors of a repairable parallel-series system with independent, non-identical and repairable components. Two types of availability equivalence factors will be discussed. These two types are referred as availability equivalent reducing factor and availability equivalent increasing factor. Following the definition of reliability equivalence factors introduced in [1]. 


\subsection{Availability Equivalence Reducing Factor}

Availability equivalence reducing factor, in short AERF, referred as $\rho=\rho_{R, B}^{D}, D=H, W, C$ for hot, warm and cold, respectively, is defined as the factor $\rho$ by which the failure rate of a set $R$ components should be reduced in order to get equality of the availability of another better design which can be obtained from the original system by assuming hot, warm and cold duplications of a set $B$ components. That is, $\rho=\rho_{R, B}^{D}$, for $D=H, W$, $C$, is the solution of the following equations in $\rho$,

$$
A_{R, \rho}=A_{B}^{D}, D=H, W, C .
$$

In what follows, we give the non-linear equations needed to be solved to get the three possible AERF's.

1) Hot availability equivalence reducing factor (HAERF): Substituting Equations (6) and (11) into Equation (18), $\rho=\rho_{R, B}^{H}$, is the solution of the following non-linear equation in $\rho$,

$$
\prod_{i=1}^{n}\left[1-\prod_{j \in R_{i}}\left(\frac{1}{1+\rho \eta_{i j}}\right) \prod_{j \in \bar{R}_{i}}\left(\frac{1}{1+\eta_{i j}}\right)\right]=\prod_{i=1}^{n}\left[1-\prod_{j \in B_{i}}\left[1-\left(\frac{\eta_{i j}}{1+\eta_{i j}}\right)^{2}\right] \prod_{j \in \bar{B}_{i}}\left(\frac{1}{1+\eta_{i j}}\right)\right] .
$$

2) Warm availability equivalence reducing factor (WAERF): Substituting Equations (6) and (14) into Equation (18), $\rho=\rho_{R, B}^{W}$, is the solution of the following non-linear equation in $\rho$,

$$
\prod_{i=1}^{n}\left[1-\prod_{j \in R_{i}}\left(\frac{1}{1+\rho \eta_{i j}}\right) \prod_{j \in \bar{R}_{i}}\left(\frac{1}{1+\eta_{i j}}\right)\right]=\prod_{i=1}^{n}\left[1-\prod_{j \in B_{i}}\left(\frac{1+\eta_{i j}+\xi_{i j}}{1+\eta_{i j}+\xi_{i j}+\frac{1}{2} \eta_{i j}^{2}+\frac{1}{2} \eta_{i j} \xi_{i j}}\right) \prod_{j \in \bar{B}_{i}}\left(\frac{1}{1+\eta_{i j}}\right)\right] .
$$

3) Cold availability equivalence reducing factor (CAERF): Substituting Equations (6) and (17) into Equation (18), $\rho=\rho_{R, B}^{C}$, satisfies the following non-linear equation

$$
\prod_{i=1}^{n}\left[1-\prod_{j \in R_{i}}\left(\frac{1}{1+\rho \eta_{i j}}\right) \prod_{j \in \bar{R}_{i}}\left(\frac{1}{1+\eta_{i j}}\right)\right]=\prod_{i=1}^{n}\left[1-\prod_{j \in B_{i}}\left(\frac{1+\eta_{i j}}{1+\eta_{i j}+\frac{1}{2} \eta_{i j}^{2}}\right) \prod_{j \in \bar{B}_{i}}\left(\frac{1}{1+\eta_{i j}}\right)\right] .
$$

Equations (19)-(21) have no closed solutions, therefore, a numerical technique method is needed to get their solutions.

\subsection{Availability Equivalence Increasing Factor}

Availability equivalence increasing factor, in short AEIF, referred as $\sigma=\sigma_{S, B}^{D}, D=H, W, C$ for hot, warm and cold, respectively, is defined as the factor $\sigma$ by which the failure rate of a set $S$ components should be reduced in order to get equality of the availability of another better design which can be obtained from the original system by assuming hot, warm and cold duplications of a set $B$ components. That is, $\sigma=\sigma_{S, B}^{D}$, for $D=H, W$, $C$, is the solution of the following equations in $\sigma$.

$$
A_{S, \sigma}=A_{B}^{D}, D=H, W, C .
$$

In what follows, we give the non-linear equations needed to be solved to get the three possible AEIF's.

1) Hot availability equivalence increasing factor (HAEIF): Substituting Equations (9) and (11) into Equation (22), $\sigma=\sigma_{S, B}^{H}$ is the solution of the following non-linear equation

$$
\prod_{i=1}^{n}\left[1-\prod_{j \in S_{i}}\left(\frac{\sigma}{\sigma+\eta_{i j}}\right) \prod_{j \in \bar{S}_{i}}\left(\frac{1}{1+\eta_{i j}}\right)\right]=\prod_{i=1}^{n}\left[1-\prod_{j \in B_{i}}\left[1-\left(\frac{\eta_{i j}}{1+\eta_{i j}}\right)^{2}\right] \prod_{j \in \bar{B}_{i}}\left(\frac{1}{1+\eta_{i j}}\right)\right] .
$$

2) Warm availability equivalence increasing factor (WAEIF): Substituting Equations (9) and (14) into Equation (22), $\sigma=\sigma_{S, B}^{W}$ is the solution of the following equation in $\sigma$

$$
\prod_{i=1}^{n}\left[1-\prod_{j \in S_{i}}\left(\frac{\sigma}{\sigma+\eta_{i j}}\right) \prod_{j \in S_{i}}\left(\frac{1}{1+\eta_{i j}}\right)\right]=\prod_{i=1}^{n}\left[1-\prod_{j \in B_{i}}\left(\frac{1+\eta_{i j}+\xi_{i j}}{1+\eta_{i j}+\xi_{i j}+\frac{1}{2} \eta_{i j}^{2}+\frac{1}{2} \eta_{i j} \xi_{i j}}\right) \prod_{j \in \bar{B}_{i}}\left(\frac{1}{1+\eta_{i j}}\right)\right] .
$$


3) Cold availability equivalence increasing factor (CAEIF): Substituting Equations (9) and (17) into Equation (22), $\sigma=\sigma_{S, B}^{C}$ is the solution of the following equation in $\sigma$,

$$
\prod_{i=1}^{n}\left[1-\prod_{j \in S_{i}}\left(\frac{\sigma}{\sigma+\eta_{i j}}\right) \prod_{j \in \bar{S}_{i}}\left(\frac{1}{1+\eta_{i j}}\right)\right]=\prod_{i=1}^{n}\left[1-\prod_{j \in B_{i}}\left(\frac{1+\eta_{i j}}{1+\eta_{i j}+\frac{1}{2} \eta_{i j}^{2}}\right) \prod_{j \in \bar{B}_{i}}\left(\frac{1}{1+\eta_{i j}}\right)\right] .
$$

The above Equations (23)-(25) have no closed-form solutions in $\sigma$, so a numerical technique method to get the value of $\sigma$.

\section{Numerical Results}

To explain how one can utilize the previously obtained theoretical results, we introduce a numerical example. In such example, we calculate the two different availability equivalence factors of a general repairable parallelseries with $n$ subsystems. Each subsystem consists of $m_{i}, i=1,2, \cdots, n$, non-identical components, under the following assumptions:

1) The parallel-series system has two subsystems, $n=2$;

2) The subsystems have the components, $m_{1}=1, m_{2}=2$ then $N=m_{1}+m_{2}=3$;

3) The values of the system parameters $\lambda_{i j}, \mu_{i j}$, and $v_{i j}\left(i=1,2, j=1, \cdots, m_{i}\right)$ are presented in Table 1.

The objective is to improve the repairable parallel-series system by improving the performance of some components instead of increasing the number of these components.

We give the values of availability of the original system and of the design obtained using the duplication methods for the example considered in this section.

Table 2 shows the availability of the original and improved system obtained from the original system by applying hot, warm and cold duplications using all possible set $B$ components, where $B=B_{1} \cup B_{2}$ and $\phi$ is the empty set.

From the results shown in Table 2, one can easily see that:

1) $A_{S}<A_{B}^{W}<A_{B}^{H}<A_{B}^{C}$, for all possible set $B$ components when $\lambda<v$;

2) $A_{S}<A_{B}^{H}<A_{B}^{W}<A_{B}^{C}$, for all possible set $B$ components when $\lambda>v$;

Table 1. Set values of the system parameters.

\begin{tabular}{|c|c|c|c|c|c|c|c|}
\hline \multirow{2}{*}{$i$} & \multirow{2}{*}{$j$} & \multicolumn{3}{|c|}{$\lambda<v$} & \multicolumn{3}{|c|}{$\lambda>v$} \\
\hline & & $\lambda$ & $v$ & $\mu$ & $\lambda$ & $v$ & $\mu$ \\
\hline 1 & 1 & 0.10 & 0.12 & 1.1 & 0.12 & 0.1 & 1.1 \\
\hline \multirow{2}{*}{2} & 1 & 0.11 & 0.13 & 1.2 & 0.13 & 0.11 & 1.2 \\
\hline & 2 & 0.12 & 0.14 & 1.3 & 0.14 & 0.12 & 1.3 \\
\hline
\end{tabular}

Table 2. The availability of the improved system, $A_{B}^{D}, D=H, W, C$.

\begin{tabular}{|c|c|c|c|c|c|c|c|c|c|}
\hline \multirow{2}{*}{$|B|$} & \multirow{2}{*}{$B=B_{1} \cup B_{2}$} & \multicolumn{4}{|c|}{$\lambda<v$} & \multicolumn{4}{|c|}{$\lambda>v$} \\
\hline & & $A_{s}$ & $A_{B}^{H}$ & $A_{B}^{W}$ & $A_{B}^{C}$ & $A_{s}$ & $A_{B}^{H}$ & $A_{B}^{W}$ & $A_{B}^{C}$ \\
\hline \multirow{4}{*}{1} & $B_{1}=\{1\}, \quad B_{2}=\phi$ & & 0.99888 & 0.99879 & 0.99939 & & 0.99821 & 0.99833 & 0.99901 \\
\hline & $B_{1}=\phi, \quad B_{2}=\{1\}$ & & 0.99242 & 0.99238 & 0.99267 & & 0.98959 & 0.98964 & 0.98997 \\
\hline & $B_{1}=\phi, \quad B_{2}=\{2\}$ & & 0.99246 & 0.99242 & 0.99271 & & 0.98955 & 0.98960 & 0.98992 \\
\hline & $B_{1}=\{1\}, \quad B_{2}=\{1\}$ & 0.98655 & 0.99937 & 0.99931 & 0.99967 & 0.98176 & 0.99898 & 0.99905 & 0.99946 \\
\hline \multirow[t]{2}{*}{2} & $B_{1}=\{1\}, \quad B_{2}=\{2\}$ & & 0.99936 & 0.99932 & 0.99967 & & 0.99897 & 0.99905 & 0.99945 \\
\hline & $B_{1}=\phi, \quad B_{2}=\{1,2\}$ & & 0.99882 & 0.99874 & 0.99936 & & 0.99814 & 0.99825 & 0.99897 \\
\hline 3 & $B_{1}=\{1\}, \quad B_{2}=\{1,2\}$ & & 0.99990 & 0.99989 & 0.99997 & & 0.99982 & 0.99984 & 0.99994 \\
\hline
\end{tabular}


3) Improving the only one component in subsystem 1, according to the duplication method, provides a better design than that can be achieved by improving one component from the subsystem 2, according to the same method;

4) Duplicating two components, one from each subsystem, produces a better design than that can be obtained by duplicating the two components in subsystem 2, according to the same method; and

5) Cold duplicating all components in the system provides the best design, in the sense of having the highest availability.

We used Mathematica Program System to calculate all possible availability equivalence factors of the studied system. Table 3 and Table 4 give the hot, warm and cold $(D=H, W, C)$ availability equivalence reducing factors, $\rho=\rho_{R, B}^{D}$, and the hot, warm and cold availability equivalence increasing factors, $\sigma=\sigma_{S, B}^{D}$, respectively, for all possible sets $R, S$ and $B$.

From the results presented in Table 3, Table 4, we can immediately conclude that:

$$
\text { Table 3. The AERF }\left(\rho_{R, B}^{D}, \quad D=H, W, C\right) \text { for different } R, B \text {, when } \lambda<v \text {. }
$$

\begin{tabular}{|c|c|c|c|c|c|c|c|c|}
\hline \multirow[b]{2}{*}{$|R|$} & \multirow[b]{2}{*}{$R=R_{1} \cup R_{2}$} & \multicolumn{3}{|c|}{$|B|=1$} & \multicolumn{3}{|c|}{$|B|=2$} & \multirow{2}{*}{$\begin{array}{c}|B|=3 \\
B_{1}=\{1\}, \\
B_{2}=\{1,2\}\end{array}$} \\
\hline & & $\begin{array}{l}B_{1}=\{1\} \\
B_{2}=\phi\end{array}$ & $\begin{array}{l}B_{1}=\phi \\
B_{2}=\{1\}\end{array}$ & $\begin{array}{l}B_{1}=\phi \\
B_{2}=\{2\}\end{array}$ & $\begin{array}{l}B_{1}=\{1\} \\
B_{2}=\{1\}\end{array}$ & $\begin{array}{l}B_{1}=\{1\} \\
B_{2}=\{2\}\end{array}$ & $\begin{array}{l}B_{1}=\phi \\
B_{2}=\{1,2\}\end{array}$ & \\
\hline \multicolumn{9}{|c|}{$\rho_{R, B}^{H}$} \\
\hline \multirow{4}{*}{1} & $R_{1}=\{1\}, \quad R_{2}=\phi$ & 0.07692 & 0.54214 & 0.53933 & 0.04323 & 0.04301 & 0.08092 & 0.00670 \\
\hline & $R_{1}=\phi, \quad R_{2}=\{1\}$ & NA & 0.07746 & 0.07202 & NA & NA & NA & NA \\
\hline & $R_{1}=\phi, \quad R_{2}=\{2\}$ & NA & 0.08333 & 0.07792 & NA & NA & NA & NA \\
\hline & $R_{1}=\{1\}, \quad R_{2}=\{1\}$ & 0.13082 & 0.64953 & 0.64706 & 0.07696 & 0.07660 & 0.13692 & 0.01264 \\
\hline \multirow[t]{2}{*}{2} & $R_{1}=\{1\}, \quad R_{2}=\{2\}$ & 0.13139 & 0.65015 & 0.64767 & 0.07735 & 0.07699 & 0.13751 & 0.01272 \\
\hline & $R_{1}=\phi, \quad R_{2}=\{1,2\}$ & 0.07384 & 0.53094 & 0.52811 & 0.04144 & 0.04123 & 0.07769 & 0.00641 \\
\hline \multirow[t]{3}{*}{3} & $R_{1}=\{1\}, \quad R_{2}=\{1,2\}$ & 0.26678 & 0.72971 & 0.72775 & 0.19877 & 0.19826 & 0.27378 & 0.07731 \\
\hline & & & & $\rho_{R, B}^{W}$ & & & & \\
\hline & $R_{1}=\{1\}, \quad R_{2}=\phi$ & 0.08333 & 0.54518 & 0.54214 & 0.04707 & 0.04682 & 0.08677 & 0.00777 \\
\hline \multirow[t]{3}{*}{1} & $R_{1}=\phi, \quad R_{2}=\{1\}$ & NA & 0.08333 & 0.07746 & NA & NA & NA & NA \\
\hline & $R_{1}=\phi, \quad R_{2}=\{2\}$ & NA & 0.08916 & 0.08333 & NA & NA & NA & NA \\
\hline & $R_{1}=\{1\}, \quad R_{2}=\{1\}$ & 0.14058 & 0.65219 & 0.64953 & 0.08333 & 0.08292 & 0.14577 & 0.01464 \\
\hline \multirow[t]{2}{*}{2} & $R_{1}=\{1\}, \quad R_{2}=\{2\}$ & 0.14118 & 0.65280 & 0.65015 & 0.08375 & 0.08333 & 0.14638 & 0.01473 \\
\hline & $R_{1}=\phi, \quad R_{2}=\{1,2\}$ & 0.08002 & 0.53398 & 0.53094 & 0.04513 & 0.04489 & 0.08333 & 0.00744 \\
\hline \multirow[t]{3}{*}{3} & $R_{1}=\{1\}, \quad R_{2}=\{1,2\}$ & 0.27793 & 0.73182 & 0.72971 & 0.20758 & 0.20702 & 0.28375 & 0.08333 \\
\hline & & & & $\rho_{R, B}^{C}$ & & & & \\
\hline & $R_{1}=\{1\}, \quad R_{2}=\phi$ & 0.04167 & 0.52376 & 0.52071 & 0.02269 & 0.02256 & 0.04394 & 0.00198 \\
\hline \multirow[t]{3}{*}{1} & $R_{1}=\phi, \quad R_{2}=\{1\}$ & NA & 0.04198 & 0.03612 & NA & NA & NA & NA \\
\hline & $R_{1}=\phi, \quad R_{2}=\{2\}$ & NA & 0.04808 & 0.04225 & NA & NA & NA & NA \\
\hline & $R_{1}=\{1\}, \quad R_{2}=\{1\}$ & 0.07435 & 0.63329 & 0.63058 & 0.04168 & 0.04146 & 0.07814 & 0.00377 \\
\hline \multirow[t]{2}{*}{2} & $R_{1}=\{1\}, \quad R_{2}=\{2\}$ & 0.07473 & 0.63393 & 0.63122 & 0.04191 & 0.04169 & 0.07854 & 0.00380 \\
\hline & $R_{1}=\phi, \quad R_{2}=\{1,2\}$ & 0.03994 & 0.51251 & 0.50946 & 0.02173 & 0.02161 & 0.04212 & 0.00190 \\
\hline 3 & $R_{1}=\{1\}, \quad R_{2}=\{1,2\}$ & 0.19508 & 0.71682 & 0.71466 & 0.14323 & 0.14283 & 0.20043 & 0.04189 \\
\hline
\end{tabular}


Table 4. The AEIF $\left(\sigma_{S, B}^{D}, D=H, W, C\right)$ for different $S, B$, when $\lambda<v$.

\begin{tabular}{|c|c|c|c|c|c|c|c|c|}
\hline \multirow[b]{2}{*}{$|S|$} & \multirow[b]{2}{*}{$S=S_{1} \cup S_{2}$} & \multicolumn{3}{|c|}{$|B|=1$} & \multicolumn{3}{|c|}{$|B|=2$} & \multirow{2}{*}{$\begin{array}{l}|B|=3 \\
B_{1}=\{1\}, \\
B_{2}=\phi\end{array}$} \\
\hline & & $\begin{array}{l}B_{1}=\{1\} \\
B_{2}=\phi\end{array}$ & $\begin{array}{l}B_{1}=\phi \\
B_{2}=\{1\}\end{array}$ & $\begin{array}{l}B_{1}=\phi \\
B_{2}=\{2\}\end{array}$ & $\begin{array}{l}B_{1}=\{1\} \\
B_{2}=\phi\end{array}$ & $\begin{array}{l}B_{1}=\phi \\
B_{2}=\{1\}\end{array}$ & $\begin{array}{l}B_{1}=\phi \\
B_{2}=\{2\}\end{array}$ & \\
\hline \multicolumn{9}{|c|}{$\sigma_{S, B}^{H}$} \\
\hline \multirow{4}{*}{1} & $S_{1}=\{1\}, \quad S_{2}=\phi$ & 13.0000 & 1.8445 & 1.8542 & 23.1343 & 23.2500 & 12.3580 & 149.2960 \\
\hline & $S_{1}=\phi, \quad S_{2}=\{1\}$ & NA & 12.9091 & 13.8852 & NA & NA & NA & NA \\
\hline & $S_{1}=\phi, S_{2}=\{2\}$ & $\mathrm{N}$ & 12.0000 & 12.8333 & $\mathrm{~N}$ & $\mathrm{~N}$ & $\mathrm{~N}$ & $\mathrm{~N}$ \\
\hline & $S_{1}=\{1\}, S_{2}=\{1\}$ & 7.6442 & 1.5396 & 1.5455 & 12.9940 & 13.0549 & 7.3034 & 79.1162 \\
\hline \multirow[t]{2}{*}{2} & $S_{1}=\{1\}, S_{2}=\{2\}$ & 7.6110 & 1.5381 & 1.5440 & 12.9283 & 12.9888 & 7.2722 & 78.6321 \\
\hline & $S_{1}=\phi, \quad S_{2}=\{1,2\}$ & NA & NA & NA & NA & NA & NA & NA \\
\hline \multirow[t]{3}{*}{3} & $S_{1}=\{1\}, \quad S_{2}=\{1,2\}$ & 3.7485 & 1.3704 & 1.3741 & 5.0310 & 5.0438 & 3.6525 & 12.9354 \\
\hline & & & & $\sigma_{s, B}^{W}$ & & & & \\
\hline & $S_{1}=\{1\}, \quad S_{2}=\phi$ & 12.0000 & 1.8343 & 1.8445 & 21.2464 & 21.3602 & 11.5245 & 128.6470 \\
\hline \multirow[t]{3}{*}{1} & $S_{1}=\phi, \quad S_{2}=\{1\}$ & NA & 12.0000 & 12.9091 & NA & NA & NA & NA \\
\hline & $S_{1}=\phi, \quad S_{2}=\{2\}$ & NA & 11.2152 & 12.0000 & NA & NA & NA & NA \\
\hline & $S_{1}=\{1\}, S_{2}=\{1\}$ & 7.1132 & 1.5333 & 1.5396 & 12.0000 & 12.0600 & 6.8604 & 68.3015 \\
\hline \multirow[t]{2}{*}{2} & $S_{1}=\{1\}, S_{2}=\{2\}$ & 7.0831 & 1.5319 & 1.5381 & 11.9404 & 12.0000 & 6.8318 & 67.8861 \\
\hline & $S_{1}=\phi, \quad S_{2}=\{1,2\}$ & NA & NA & NA & NA & NA & NA & NA \\
\hline \multirow[t]{3}{*}{3} & $S_{1}=\{1\}, \quad S_{2}=\{1,2\}$ & 3.5980 & 1.3665 & 1.3704 & 4.8174 & 4.8305 & 3.5242 & 12.0000 \\
\hline & & & & $\sigma_{s, B}^{C}$ & & & & \\
\hline & $S_{1}=\{1\}, \quad S_{2}=\phi$ & 24.0000 & 1.9093 & 1.9205 & 44.0802 & 44.3267 & 22.7607 & 504.5480 \\
\hline \multirow[t]{3}{*}{1} & $S_{1}=\phi, \quad S_{2}=\{1\}$ & NA & 23.8182 & 27.6840 & NA & NA & NA & NA \\
\hline & $S_{1}=\phi, \quad S_{2}=\{2\}$ & NA & 20.7991 & 23.6667 & NA & NA & NA & NA \\
\hline & $S_{1}=\{1\}, S_{2}=\{1\}$ & 13.4495 & 1.5791 & 1.5859 & 23.9930 & 24.1223 & 12.7973 & 265.1530 \\
\hline \multirow[t]{2}{*}{2} & $S_{1}=\{1\}, S_{2}=\{2\}$ & 13.3809 & 1.5775 & 1.5842 & 23.8585 & 23.9870 & 12.7328 & 263.4860 \\
\hline & $S_{1}=\phi, \quad S_{2}=\{1,2\}$ & NA & NA & NA & NA & NA & NA & NA \\
\hline 3 & $S_{1}=\{1\}, \quad S_{2}=\{1,2\}$ & 5.1260 & 1.3951 & 1.3993 & 6.9816 & 7.0014 & 4.9894 & 23.8707 \\
\hline
\end{tabular}

1) Hot duplication of the only one component in subsystem $1, B_{1}=\{1\}$ and $B_{2}=\phi$ increases the system availability from $A_{S}=0.98655$ to $A_{B}^{H}=0.99888, B=B_{1} \cup B_{2}$, see Table 2 . The improved system with $A_{B}^{H}=$ 0.99888 can be achieved by performing one of the following:

a) Reducing the failure rate(s) of (see Table 3): i) the only component in subsystem $1, \quad R=R_{1} \cup R_{2}$, where $R_{1}=\{1\}$ and $R_{2}=\phi$, by the HAERF $\rho_{R, B}^{H}=0.07692$, ii) the only component in subsystem 1 and the first component in subsystem $2, R_{1}=\{1\}, R_{2}=\{1\}$, by the HAERF $\rho_{R, B}^{H}=0.13082$, iii) the only component in subsystem 1 and the second component in subsystem $2, R_{1}=\{1\}, R_{2}=\{2\}$, by the HAERF $\rho_{R, B}^{H}=0.13139$, iv) the two components in subsystem $2, R_{1}=\phi$ and $R_{2}=\{1,2\}$, by the HAERF $\left.\rho_{R, B}^{H}=0.07384, v\right)$ all the three components, $R_{1}=\{1\}, R_{2}=\{1,2\}$, by the HAERF $\rho_{R, B}^{H}=0.26678$.

b) Increasing the repair rate(s) of (see Table 4): i) the only component in subsystem $1, S=S_{1} \cup S_{2}$, where $S_{1}=\{1\}$ and $S_{2}=\phi$, by the HAEIF $\sigma_{S, B}^{H}=13.0000$, ii) the only component in subsystem 1 and first compo- 
nent in subsystem $2, S_{1}=\{1\}, S_{2}=\{1\}$, by the HAEIF $\sigma_{S, B}^{H}=7.6442$, iii) the only component in subsystem 1 and second component in subsystem $2, S_{1}=\{1\}, S_{2}=\{2\}$, by the HAEIF $\sigma_{S, B}^{H}=7.6110$, iv) all the three components, $S_{1}=\{1\}, S_{2}=\{1,2\}$, by the HAEIF $\sigma_{S, B}^{H}=3.7485$.

2) Warm duplication of the only component in subsystem $1, B_{1}=\{1\}$ and $B_{2}=\phi$, increases the system availability from $A_{S}=0.98655$ to $A_{B}^{W}=0.99879, B=B_{1} \cup B_{2}$ see Table 2 . The improved system with $A_{B}^{W}=$ 0.99879, can be achieved by performing one of the following:

a) Reducing the failure rate(s) of (see Table 3): i) the only component in subsystem 1, $R=R_{1} \cup R_{2}$ where $R_{1}=\{1\}$ and $R_{2}=\phi$, by the WAERF $\rho_{R, B}^{W}=0.08333$, ii) the only component in subsystem 1 and the first component of subsystem $2, R_{1}=\{1\}, R_{2}=\{1\}$, by the WAERF $\rho_{R, B}^{W}=0.14058$, iii) the only component in subsystem 1 and the second component of subsystem $2, R_{1}=\{1\}, R_{2}=\{1\}$, by the WAERF $\rho_{R, B}^{W}=0.14118$, iv) the two components in subsystem $2, R_{1}=\phi, R_{2}=\{1,2\}$, by the WAERF $\rho_{R, B}^{W}=0.08002$, v) all three components, $R_{1}=\{1\}, R_{2}=\{1,2\}$, by the WAERF $\rho_{R, B}^{W}=0.27793$.

b) Increasing the repair rate(s) of (see Table 4): i) the only component in subsystem $1, \quad S=S_{1} \cup S_{2}$ where $S_{1}=\{1\}$ and $S_{2}=\phi$, by the WAEIF $\sigma_{S, B}^{W}=12.0000$, ii) the only component in subsystem 1 and first component of subsystem $2, S_{1}=\{1\}, S_{2}=\{1\}$, by the WAEIF $\sigma_{S, B}^{W}=7.1132$, iii) the only component in subsystem 1 and second component of subsystem $2, S_{1}=\{1\}, S_{2}=\{2\}$, by the WAEIF $\sigma_{S, B}^{W}=7.0831$, iv) all three components, $S_{1}=\{1\}, S_{2}=\{1,2\}$, by the WAEIF $\sigma_{S, B}^{W}=3.5980$.

3) Cold duplication of the only component in subsystem $1, B_{1}=\{1\}$ and $B_{2}=\phi$, increases the system availability from $A_{s}=0.98655$ to $A_{B}^{C}=0.99939$, see Table 2 . The improved system with $A_{B}^{C}=0.99939$, can be achieved by performing one of the following:

a) Reducing the failure rate(s) of (see Table 3): i) the only component in subsystem $1, R=R_{1} \cup R_{2}$ where $R_{1}=\{1\}$ and $R_{2}=\phi$ by the CAERF $\rho_{R, B}^{C}=0.04167$, ii) the only component in subsystem 1 and first component of subsystem $2, R_{1}=\{1\}, R_{2}=\{1\}$, by the CAERF $\rho_{R, B}^{C}=0.07435$, iii) the only component in subsystem 1 and second component of subsystem $2, R_{1}=\{1\}, R_{2}=\{2\}$, by the CAERF $\rho_{R, B}^{C}=0.07473$, iv) the two components in subsystem $2, R_{1}=\phi, R_{2}=\{1,2\}$, by the CAERF $\rho_{R, B}^{C}=0.03994$, v) all three components, $R_{1}=\{1\}$, $R_{2}=\{1,2\}$, by the CAERF $\rho_{R, B}^{C}=0.19508$.

b) Increasing the repair rate(s) of (see Table 4): i) the only component in subsystem $1, \quad S=S_{1} \cup S_{2}$ where $S_{1}=\{1\}$ and $S_{2}=\phi$ by the CAEIF $\sigma_{S, B}^{C}=24.0000$, ii) the only component in subsystem 1 and first component of subsystem $2, S_{1}=\{1\}, S_{2}=\{1\}$, by the CAEIF $\sigma_{S, B}^{C}=13.4495$, iii) the only component in subsystem 1 and second component of subsystem $2, S_{1}=\{1\}, S_{2}=\{2\}$, by the CAEIF $\sigma_{S, B}^{C}=13.3809$, iv) all three components, $S_{1}=\{1\}, S_{2}=\{1,2\}$, by the CAEIF $\sigma_{S, B}^{C}=5.1260$.

4) In the same manner, we can illustrate the rest of results shown in Table 3 and Table 4.

5) The notation NA, means that there is no possible equivalence between the two improved systems that can be achieved by reducing (increasing) the failure (repair) rates of the set $R(S)$ of system components and that can be achieved by duplicating elements of set $B$ of system components.

\section{Conclusions}

This paper discusses the availability equivalence factors of a general repairable parallel-series system with independent but non-identical components. The system studied here generalizes several well-known systems such as a repairable parallel-series system with independent and identical components; repairable series and repairable parallel systems with independent and non-identical or identical components. We derived two types of the availability equivalence factors of the system. We presented a numerical example to illustrate how the theoretical results derived in the paper can be applied.

Indeed there are several possible extensions of this work. As an example, the case of a general repairable parallel-series system with non-constant failure rates can be studied.

\section{References}

[1] Sarhan, A.M. (2002) Reliability Equivalence with a Basic Series/Parallel System. Applied Mathematics and Computation, 132, 115-133. http://dx.doi.org/10.1016/S0096-3003(01)00181-3

[2] Sarhan, A.M. (2009) Reliability Equivalence Factors of a General Series-Parallel System. Reliability Engineering and System Safety, 94, 229-236. http://dx.doi.org/10.1016/j.ress.2008.02.021

[3] Sarhan, A.M., Al-Ruzaiza, A.S., Alwasel, I.A. and El-Gohary, A.I. (2004) Reliability Equivalence of a Series-Parallel 
System. Applied Mathematics and Computation, 154, 257-277. http://dx.doi.org/10.1016/S0096-3003(03)00709-4

[4] Råde, L. (1993) Reliability Equivalence. Microelectronics Reliability, 33, 323-325. http://dx.doi.org/10.1016/0026-2714(93)90020-Y

[5] Meng, F.C. (1993) On Selecting Components for Redundancy Incoherent Systems. Reliability Engineering and System Safety, 41, 121-126. http://dx.doi.org/10.1016/0951-8320(93)90025-T

[6] Mustafa, A. (2009) Reliability Equivalence Factor of n-Components Series System with Non-Constant Failure Rates. International Journal of Reliability and Applications, 10, 43-58.

[7] Hu, L., Yue, D. and Zhao, D. (2012) Availability Equivalence Analysis of a Repairable Series-Parallel System. Mathematical Problems in Engineering, 2012, Article ID: 957537. http://dx.doi.org/10.1155/2012/957537

[8] Sarhan, A.M. and Mustafa, A. (2013) Availability Equivalence Factors of a General Repairable Series-Parallel System. International Journal of Reliability and Applications, 14, 11-26.

[9] Lewis, E.E. (1996) Introduction to Reliability Engineering. 2nd Edition, Wiley, New York.

[10] Leemis, L.M. (1996) Reliability Probabilistic Models and Statistical Methods. Prentice-Hall, Englewood Cliffs.

[11] Kumar, S., Chattopadhyayb, G. and Kumar, U. (2007) Reliability Improvement through Alternative Designs-A Case Study. Reliability Engineering and System Safety, 92, 983-991. http://dx.doi.org/10.1016/j.ress.2006.05.008

[12] Billinton, R. and Wang, P. (1999) Deregulated Power System Planning Using a Reliability Network Equivalent Technique. IEE Proceedings-Generation, Transmission and Distribution, 146, 25-30. http://dx.doi.org/10.1049/ip-gtd:19990046

[13] Sarhan, A.M., Tadj, L., Al-Khedhairi, A. and Mustafa, A. (2008) Equivalence Factors of a Parallel-Series System. Applied Sciences, 10, 219-230.

[14] Xia, Y. and Zhang, G. (2007) Reliability Equivalence Factors in Gamma Distribution. Applied Mathematics and Computation, 187, 567-573. http://dx.doi.org/10.1016/j.amc.2006.07.016

[15] Mustafa, A. and El-Bassoiuny, A.H. (2009) Reliability Equivalence of Some Systems with Mixture Linear Increasing Failure Rates. Pakistan Journal of Statistics, 25, 149-163.

[16] Mustafa, A. and El-Faheem, A.A. (2012) Reliability Equivalence Factors of a General Parallel System with Mixture Lifetimes. Applied Mathematical Sciences, 6, 3769-3784.

[17] Mustafa, A., Sarhan, A.M. and Al-Ruzaiza, A.S. (2007) Reliability Equivalence of a Parallel-Series System. Pakistan Journal of Statistics, 23, 241-254.

[18] Wang, Z.H. (1992) Reliability Engineering Theory and Practice. Quality Control Society of Republic of China, Taipei, China.

[19] Juang, Y.S., Lin, S.S. and Kao, H.P. (2008) A Knowledge Management System for Series-Parallel Availability Optimization and Design. Expert Systems with Applications, 34, 181-193. http://dx.doi.org/10.1016/j.eswa.2006.08.023

[20] Kolowrocki, K. (1994) Limit Reliability Functions of Some Series-Parallel and Parallel-Series Systems. Applied Mathematics and Computation, 62, 129-151. http://dx.doi.org/10.1016/0096-3003(94)90078-7

[21] Liu, Y. and Zheng, H. (2010) Study on Reliability of Warm Standby’s Repairable System with n Identity Units and k Repair Facilities. Journal of Wenzhou University, 31, 24-29.

[22] Gu, J. and Wei, Y. (2006) Reliability Quantities of a n-Unit Cold Standby Repairable System with Two Repair Facility. Journal of Gansu Lianhe University, 20, 17-20. 